\title{
Dimensionally Renormalized Green's Functions for Theories with Massless Particles. I.
}

\author{
P. Breitenlohner and D. Maison* \\ Max-Planck-Institut für Physik und Astrophysik, D-8000 München 40, Federal Republic of Germany
}

\begin{abstract}
In the framework of dimensional renormalization the existence of Green's functions to all orders of perturbation theory is proved for theories of massless particles without super-renormalizable couplings. For those Green's functions Schwinger's Action Principle holds as in the massive case.
\end{abstract}

\section{Introduction}

In a previous publication [1], an attempt was made to give a consistent formulation of the socalled "Dimensional Renormalization" to all orders of perturbation theory such that Schwinger's Action Principle holds. That was done under the provision that all particles were massive. In the present paper we want to relax this condition. More precisely we shall treat here only the case that all particles are massless and the theory contains no interactions of super-renormalizable type. In a subsequent publication we shall come to the general case of both massive and massless particles, which is complicated by the fact that additional finite subtractions for subgraphs with positive superficial degree of divergence have to be made in order to guarantee their correct normalization.

In contrast to the BPHZ method the Action Principle holds unmodified by radiative corrections in almost all cases discussed in the literature, with the exception of the few occasions where these corrections are known to be unavoidable (e.g. Trace identities, Adler-anomaly) ${ }^{1}$.

The physical relevance of a renormalization scheme that allows the treatment of massless particles on one hand and in which the Action Principle holds on the other is obvious, especially in view of theories with gauge invariance of the second kind. We want to emphasize, however, that we do not tackle the physical infrared problem here, i.e. the definition of the $S$-matrix for massless particles.

* Present address: II. Institut für Theoretische Physik der Universität Hamburg, Luruper Chaussee 149, D-2000 Hamburg 50, Federal Republic of Germany

1 The discussion of super-symmetries is still missing in this framework 
Dimensional renormalization as outlined in [1] proceeds in two steps:

i) Dimensionally regularized Feynman amplitudes are defined by treating the parameter $n$ of space-time dimension as a regularizing device; a unique prescription is given to extract the $n$-dependence of Lorentz covariants like spin polynomials etc.; these regularized Feynman amplitudes turn out to be distributions which are meromorphic functions of $n$.

ii) The poles of these meromorphic functions are eliminated in a way consistent with additive renormalization.

The regularized Feynman amplitudes are defined via their Feynman parameter integral representation:

$$
\mathscr{T}_{G}(\underline{p}, n)=c_{G} \delta\left(\sum p_{i}\right) \int_{0}^{\infty} \prod_{\mathscr{L}_{G}} d \alpha_{\ell} I_{G}(p, \underline{\alpha}, n)
$$

where $I_{G}(\underline{p}, \underline{\alpha}, n)$ is a distribution in $\underline{p}$, depending parametrically on $\underline{\alpha}$ and $n$.

The UV divergencies turn up as non-integrable singularities of the integrand when certain subsets of $\alpha$ 's vanish. The nature of these singularities can be displayed by a subdivision of the domain of integration and the introduction of suitable "scaling variables" in place of the $\alpha$ 's [2].

In the case of massless particles the integral may also diverge when certain $\alpha$ 's tend to infinity. These IR singularities can be analyzed by the same device [3].

Once this has been established it is just a matter of power counting to show that in the absence of super-renormalizable interactions no such singularities of the infrared type are encountered in a neighbourhood of $n=4$ as long as $\mathscr{T}_{G}(p, n)$ is considered as a distribution in $p$ (i.e. there are clearly physical region singularities, like in the massive case).

The subsequent subtraction of the UV poles in $n$, i.e. the renormalization proceeds like in the massive case [1]. Also in the proof of the Action Principle there is no change.

\section{Dimensionally Regularized Feynman Amplitudes}

\section{II.1. Analysis of the Singularity Structure}

As in the massive case, treated in [1], we start from the formal Feynman parameter integral representation for the amplitude corresponding to some connected graph $G$ with $h_{G}$ loops

$$
\begin{aligned}
i \mathscr{T}_{G}\left(p_{1}, \ldots, p_{M}, n\right)= & (2 \pi)^{n / 2} \delta\left(\sum_{i=1}^{M} p_{i}\right)(i \hbar)^{h_{G}-1}(2 i)^{-(n / 2) h_{G}} \\
& \cdot \lim _{\varepsilon \rightarrow 0} \int_{0}^{\infty} \prod_{\mathscr{L}_{G}} d \alpha_{\ell}\left[\left.I_{G, \varepsilon}(p, \underline{u}, \underline{\alpha}, n)\right|_{\underline{u}=0}\right]
\end{aligned}
$$

where

$$
I_{G, \varepsilon}(p, \underline{u}, \underline{\alpha}, n)=d(\alpha)^{-n / 2} \prod_{\mathscr{L}_{G}} Z_{\ell}\left(-i \partial / \partial u_{\ell}\right) \exp \left[i V(\underline{p}, \underline{u}, \underline{\alpha})-\varepsilon \sum_{\mathscr{L}_{G}} \alpha_{\ell}\right],
$$

the quadratic form $V$ is given by

$$
V(\underline{p}, \underline{u}, \underline{\alpha})=\left(\underline{p}^{+}, \underline{u}^{+}\right)\left(\begin{array}{cc}
0 & -2 e_{E}^{+} \\
-2 e_{E} & -4 \alpha
\end{array}\right)^{-1}\left(\begin{array}{l}
\underline{p} \\
\underline{u}
\end{array}\right)
$$

and $p=\left(p_{1}, \ldots, \hat{p}_{k}, \ldots, p_{M}\right)$ (cf. [1]). 
As was outlined in [1] the execution of the $Z_{\ell}$ 's has to produce a certain $n$-dependence of $I_{G, \varepsilon}$ in order that the Action Principle holds. An abstract algebra of covariants was therefore developed allowing to extend certain algebraic relations between 4-dimensional Feynman amplitudes valid for tree graphs to graphs with any number of loops.

$I_{G, \varepsilon}(\underline{p}, \underline{u}, \underline{\alpha}, n)$ is first considered as a member of this abstract algebra and then reduced to its normal form (NF) in order to make its $n$-dependence explicit. The resulting expression can be interpreted as a distribution in 4-dimensional space depending parametrically on $n$.

The next step to be performed is the limit $\varepsilon \rightarrow 0$.

Up to now we have not specified which are the internal and which the external vertices of the graph $G$, i.e. which momenta $p$ will eventually be set to zero and which we want to keep as variables. In the massive case that is unimportant since $\left(V(\underline{p}, \underline{\alpha})-\sum \alpha_{\ell} m_{\ell}^{2}+i 0\right)^{\omega}$ considered as distribution in $p$ is an entire function of $\omega$ independent of the dimension of $\underline{p}$. This is no more true if some or all of the $m_{\ell}$ 's are zero. In fact, the distribution $\left(p^{2}+i 0\right)^{\omega}$ is meromorphic in $\omega$ with poles at $\omega=-N / 2,-N / 2-1, \ldots$ where $N$ is the dimension of $p[4]$. In order to analyze the possible singularities introduced by the limit $\varepsilon \rightarrow 0$ it is therefore important to control the degeneracy of the quadratic form $V$ as a function of $\underline{\alpha}$.

For the same reasons it is non-trivial to construct the Feynman amplitudes for $1 P R$-graphs from those of their $1 P I$-components as it was possible in the massive case. Therefore we include into the subsequent consideration $1 P R-$ graphs, assume, however, for the moment that $G$ is connected and contains no tadpoles. We will come back to the question of tadpoles at the end of Section 3.

Once the expression for $I_{G, \varepsilon}$ has been brought to its NF and all $u_{\ell}$ 's as well as all auxiliary momenta $p_{i}(i>K)$ are put to zero the quadratic form $V$ takes the conventional form [2]

$$
V\left(\underline{p}^{\mathrm{ext}}, \underline{\alpha}\right)=\left.V(\underline{p}, 0, \underline{\alpha})\right|_{\underline{p}=\underline{p}^{\mathrm{ext}}}=\left.\underline{p}^{+} A_{E}^{-1} \underline{p}\right|_{\underline{p}=\underline{p} \underline{\mathrm{ext}}}=\sum_{i, j=1}^{K-1} D_{i j}(\alpha) d(\alpha)^{-1} p_{i} p_{j} .
$$

Applying a technique developed in Ref. [3] we divide the domain of integration in $\alpha$-space into sectors corresponding to labelled $c_{\infty}$-families $\left(\mathscr{C}_{\infty}, \sigma\right)$ as defined in Appendix A. In each sector $\mathscr{D}\left(\mathscr{C}_{\infty}, \sigma\right)$ given by $\left\{\underline{\alpha}: 0 \leqq \alpha_{\ell} \leqq \alpha_{\sigma(H)}\right.$ for $\ell \in \mathscr{L}_{H}$, $H \in \mathscr{C}_{\infty}$ ) we introduce "scaling variables" $t_{H}$ as well as auxiliary variables $\eta_{H}$ and $\zeta_{H}$ according to

$$
\alpha_{\sigma(H)}=\prod_{H \subset H^{\prime} \in \mathscr{C}_{\infty}} t_{H^{\prime}}^{2}=\zeta_{H}^{2}=t_{G}^{2} \eta_{H}^{2} \quad \text { for } \quad H \in \mathscr{C}_{\infty}
$$

In addition we introduce variables

$$
\beta_{\ell}=\alpha_{\ell} / \zeta_{H_{\ell}}^{2} \text { for } \ell \in \mathscr{L}_{\mathrm{G}}
$$

where $H_{\ell}$ is the smallest member of $\mathscr{C}_{\infty}$ containing line $\ell$. Some of the $\beta_{\ell}$ 's are identically one, namely those for which $\ell \in \sigma\left(\mathscr{C}_{\infty}\right)$. Instead of $\underline{\alpha}$ we take $(t, \underline{\beta})=$ $\left(t_{H}, H \in \mathscr{C}_{\infty}, \beta_{\ell}, \ell \notin \sigma\left(\mathscr{C}_{\infty}\right)\right)$ as new variables. In these new variables the sector $\mathscr{D}\left(\mathscr{C}_{\infty}, \sigma\right)$ is given by $\left\{0 \leqq t_{G}<\infty, 0 \leqq t_{H} \leqq 1\right.$ for $\left.H \neq G, 0 \leqq \beta_{\ell} \leqq 1\right\}$. 
The Feynman integral for $G$ decomposes into a sum of terms

$$
\begin{gathered}
\int_{0}^{\infty}\left(d t_{G} / t_{G}\right) t_{G}^{v h_{G}-\omega_{G}+r} \int_{0}^{1} \prod_{G \neq H \in \mathscr{C}_{\infty}}\left(d t_{H} / t_{H}\right) t_{H}^{\nu h_{H}-\omega_{H}} \int_{0}^{1} \prod^{\prime} d \beta_{\ell} \\
\cdot g_{r}(p, \underline{t}, \underline{\beta}, n) \exp i t_{G}^{2}\left[\hat{V}(p, \underline{\underline{t}}, \underline{\beta})+i \varepsilon \sum_{\mathscr{L}_{G}} \beta_{\ell} \eta_{H_{\ell}}^{2}\right]
\end{gathered}
$$

where the following notations are employed: $\prod^{\prime} d \beta_{\ell}$ denotes the product over the subset of $\mathscr{L}_{G}$, for which $\beta_{\ell} \neq 1, v=4-n$ and $\omega_{G}$ resp. $\omega_{H}$ is the superficial degree of divergence of $G$ resp. $H ; g_{r}$ is a homogeneous polynomial in $p$ of degree $r$ with coefficients which are $C^{\infty}$ in $(t, \beta)$ and holomorphic in $n$.

$g_{r}$ as well as the expression in square brackets are independent of $t_{G}$. Therefore we can perform the integral over $t_{G}$ [absolutely convergent for $\varepsilon>0$ and $\operatorname{Re}(v) \gg 0$ ] with the result:

$$
\begin{gathered}
\Gamma\left(\left(v h_{G}-\omega_{G}+r\right) / 2\right) \int_{0}^{1} \prod_{H \neq G}\left(d t_{H} / t_{H}\right) t_{H}^{v h_{H}-\omega_{H}} \int_{0}^{1} \prod^{\prime} d \beta_{\ell} g_{r}(\underline{p}, \underline{t}, \underline{\beta}, n) \\
\cdot\left[-i \hat{V}(\underline{p}, \underline{t}, \underline{\beta})+\varepsilon \sum_{\mathscr{L}_{G}} \beta_{\ell} \eta_{H}^{2}\right]^{\left(\omega_{G}-v h_{G}-r\right) / 2} .
\end{gathered}
$$

As demonstrated in Appendix A, the quadratic form $\hat{V}$ can be brought to a form explicitly displaying its possible degeneracy. This is achieved by taking certain linear combinations $q_{H}$ [independent of $\left.(t, \underline{\beta})\right]$ of the external momenta $p_{i}$ as new variables. More precisely

$$
\hat{V}(p, \underline{t}, \underline{\beta})=\sum_{\boldsymbol{H}, \boldsymbol{H}^{\prime} \in \mathscr{H}} d_{H H^{\prime}} \eta_{H} \eta_{H^{\prime}} q_{H} q_{\boldsymbol{H}^{\prime}}
$$

with $\left\{d_{H H^{\prime}}(t, \underline{\beta})\right\}_{H, H^{\prime} \in \mathscr{H}}$ a positive definite matrix which is $C^{\infty}$ in $(t, \underline{\beta})$ from the domain $\mathscr{D}\left(\mathscr{C}_{\infty}, \sigma\right)$. Therefore the whole degeneracy of the quadratic form $\hat{V}$ is exhibited by the $\eta_{H}$ 's. The possible singularities resulting from such a degeneracy of the quadratic form can, however, be controlled by the following

\&-Lemma. Let $\left\{\eta_{j}, \varrho_{j}\right\}_{j=1}^{J}$ and $\left\{\gamma_{\ell}, k_{\ell}, x_{\ell}\right\}_{\ell=1}^{L}$ be real numbers in the domain:

$$
\begin{aligned}
& 0 \leqq \eta_{J} \leqq \ldots \leqq \eta_{1} ; \quad 0<\varrho_{j}<4 \text { for } j=1, \ldots, J ; \\
& \gamma_{\ell} \geqq 0 \text { and } k_{\ell}, x_{\ell}>0 \text { for } \ell=1, \ldots, L .
\end{aligned}
$$

In addition take $\varrho \in \mathbb{C},\left\{d_{i j}\right\}_{i, j=1}^{J}$ a positive definite matrix defining the quadratic form $d(q)=\sum_{i, j=1}^{J} d_{i j} q_{i} q_{j}$ over $J$ copies of 4-dimensional Minkowski space and $Q(q)$ a homogeneous polynomial of degree $r$ in $\underline{q}$.

$$
\begin{aligned}
& \text { Then for } \operatorname{Re}(\varrho)<\sum_{j=1}^{J} \varrho_{j}+2 \sum_{\ell=1}^{L} k_{\ell}+r \\
& Q\left(\eta_{j} q_{j}\right) \prod \eta_{j}^{\varrho_{j}} \prod \gamma_{\ell}^{k_{\ell}}\left(-i d\left(\eta_{j} q_{j}\right)+i \sum \gamma_{\ell} x_{\ell}+0\right)^{-\varrho / 2} \\
& =\lim _{\varepsilon \rightarrow 0} Q\left(\eta_{j} q_{j}\right) \prod \eta_{j}^{\varrho_{j}} \prod \gamma_{\ell}^{k_{\ell}}\left(-i d\left(\eta_{j} q_{j}\right)+i \sum \gamma_{\ell} x_{\ell}+\varepsilon\right)^{-\varrho / 2}
\end{aligned}
$$

exists in $\mathscr{S}^{\prime}\left(\mathbb{R}^{4 J}\right)$, is continous in $(\eta, \gamma)$ and $C^{\infty}$ in the coefficients of $Q$ and $d$.

Proof. For the proof we refer to Appendix B. 
Remark. The reader may wonder why we have included masses $\varkappa_{\ell}$. This will become clear in Section 3.

In view of this lemma we rewrite Equation (8) in the.form

$$
\begin{aligned}
& \Gamma\left(\left(v h_{G}-m_{G}+r\right) / 2\right) \int_{0}^{1} \prod_{H \neq G}\left(d t_{H} / t_{H}\right) t_{H}^{\nu h_{H}-\omega_{H}-\sigma_{H}} \int_{0}^{1} \prod^{\prime} d \beta_{\ell} h(\underline{t}, \underline{\beta}, v) \\
& \cdot Q\left(\eta_{H} q_{H}\right) \prod_{\mathscr{H}} \eta_{H}^{O_{H}}\left(-i d\left(\eta_{H} q_{H}\right)+\varepsilon\right)^{-\left(v h_{G}-\omega_{G}+r\right) / 2} .
\end{aligned}
$$

According to Lemma $8 h$ is some $C^{\infty}$ function of $(t, \beta)$, entire analytic in $v ; \sigma_{H}=$ $\sum_{H \supset H^{\prime} \in \mathscr{H}} \varrho_{H^{\prime}}$ with for the moment undetermined real numbers $\varrho_{H^{*}}$. For the renormalization it is essential that we are able to choose the $\varrho_{H}$ 's in such a way that in a neighbourhood of $v=0$ no new singularities, in addition to the UV poles, are introduced by the limit $\varepsilon \rightarrow 0$. To decide under which conditions that is possible we have to investigate the distribution of the numbers $\omega_{H}$ for various subgraphs $H \in \mathscr{C}_{\infty}$.

Let $\mathscr{U}_{G}$ be the set of all external vertices of $G$. We state some well-known facts about power counting [5].

(a) Let $H \subset G$ be any connected subgraph of $G$, then a positive number $\delta_{a}^{H} \leqq \delta_{a}^{G}$ - the canonical dimension-is given for any vertex $V_{a}$ of $H$. The superficial degree of divergence of $H$ is expressed by

$$
\omega_{H}=4-\sum_{\mathscr{V}_{H}}\left(4-\delta_{a}^{H}\right) .
$$

(b) If $H$ has connectivity components $H_{i}$, then $\omega_{H}=\sum_{i} \omega_{H_{i}}$.

Definition. We split the degree $\omega_{G}$ into the internal degree of $G$ given by

$$
\omega_{G}^{\text {int }}=-\sum_{\mathscr{V}_{G} \backslash \mathscr{U}_{G}}\left(4-\delta_{a}^{G}\right)
$$

and the external degree of $G$

$$
\omega_{G}^{\mathrm{ext}}=\omega_{G}-\omega_{G}^{\mathrm{int}}
$$

For any $H \subset G$ we define the relative external degree of $H$ in $G$ by

$$
\omega_{H, G}^{\mathrm{ext}}=\sum_{i} \omega_{H_{i}, G}^{\mathrm{ext}}=\sum_{i}\left(4-\sum_{\mathscr{U}_{H_{i}}}\left(4-\delta_{a}^{G}\right)\right)
$$

where $H_{i}$ are again the $c$-components of $H$.

Under the assumption that the Lagrangean contains no super-renormalizable couplings $\omega_{G}^{\text {int }} \geqq 0$ for all graphs $G$. $\omega_{G}^{\text {int }}=0$ characterizes strictly renormalizable theories.

For the sake of clarity we divide the further discussion of the limit $\varepsilon \rightarrow 0$ into a simpler case-Green's functions for strictly renormalizable theories-and the general one. 


\section{II.2. Green's Functions for Strictly Renormalizable Interactions}

In this section we treat only graphs $G$ with $\delta_{a}^{G}=4$ resp. $\delta_{a}^{G} \leqq 3$ for all internal resp. external vertices. In order to simplify the discussion further, we consider only non-amputated Green's functions, i.e. we assume that every external vertex of $G$ is connected to the rest of the graph by exactly one line, i.e. $\delta_{a}^{G}=1$ or $3 / 2$. The amplitudes for arbitrary graphs are obtained by multiplication with a suitable polynomial in the external momenta.

In this case we get

$$
\left.\omega_{H, G}^{\mathrm{ext}}=\sum_{i}\left(4-3 b_{H_{i}, G}-5 / 2 f_{H_{i}, G}\right)\right)
$$

where $b_{H_{i}, G}$ resp. $f_{H_{i}, G}$ is the number of boson resp. fermion lines of $H_{i}$ connected to the external vertices of $G$.

Lemma 1. Under the assumptions made above we get $\omega_{H}<0$ for all $H \in \mathscr{H}$, where $\mathscr{H}$ is the $\mathscr{H}$-part of any $c_{\infty}$-family.

Proof. Every $c$-component $H_{i}$ of $H \in \mathscr{H}$ contains at least two external vertices of $G$, therefore $\omega_{H} \leqq \sum_{i} \omega_{H_{i}, G}^{\text {ext }} \leqq-1$.

After these preliminaries we can proceed to the definition of the $\sigma_{H}$ 's resp. $\varrho_{H}$ 's from Equation (11). We take some real number $a$ with $0<a<1$ and put

$$
\sigma_{H}=\max \left\{0, \sum_{i}\left(a-\omega_{H_{\imath}, G}^{\mathrm{ext}}\right)\right\} \text { for } H \in \mathscr{C}_{\infty},
$$

the sum running once more over the $c$-components of $H$. Notice that $\sigma_{H}$ is the same for all $H \in \mathscr{C}_{\infty}$ with the same $H^{-}$and $\sigma_{H}=0$ if $H^{-}$does not exist, i.e. $\left\{H^{\prime} \in \mathscr{H}: H^{\prime} \subset H\right\}=\emptyset$. We claim that this definition meets all the requirements needed for the application of the $\varepsilon$-Lemma. This is expressed by

Lemma 2. (a) $-\omega_{H}-\sigma_{H} \geqq 1-a$ for all $H \in \mathscr{C}_{\infty}, H \neq G$, which contain some $H^{\prime} \in \mathscr{H}$ (i.e. if $H^{-}$exists); otherwise $\sigma_{H}=0$;

(b) the $\varrho_{H}$ 's corresponding to the $\sigma_{H}$ 's obey $0<\varrho_{H} \leqq 4-a$;

(c) $\sum_{H \in \mathscr{H}} \varrho_{H}+r=-\omega_{G}+r+a$.

Proof. (a) For every $c$-component $H_{i}$ of $H \omega_{H_{2}} \leqq \omega_{H_{i}, G}^{\text {ext }}-1$ ( $G$ is connected) and therefore

$$
\begin{aligned}
-\omega_{H}-\sigma_{H} & =-\omega_{H}+\sum_{i}\left(\omega_{H_{i}, G}^{\mathrm{ext}}-a\right) \\
& \geqq \sum_{i}\left[-\left(\omega_{H_{i}, G}^{\mathrm{ext}}-1\right)+\left(\omega_{H_{i}, G}^{\mathrm{ext}}-a\right)\right] \geqq 1-a .
\end{aligned}
$$

(b)

$$
\varrho_{H}=\left\{\begin{array}{l}
\sigma_{H} \text { if there exists no } H_{-} ; \\
\sigma_{H}-\sigma_{H_{-}} \text {else }
\end{array}\right.
$$

For $\varrho_{H}=\sigma_{H}-\sigma_{H_{-}}$there are three possibilities (Lemma $4 \mathrm{f}$ ).

i) $H$ and $H_{-}$share the same external vertices with $G$, then $\varrho_{H}=4-a$;

ii) $H_{-}$has one of the external vertices of $G$ less than $H$, then $\varrho_{H}=3$ or $5 / 2$;

iii) $H_{-}$has two of the external vertices of $G$ less than $H$ (or $H_{-}$does not exist), 
then $\varrho_{H}=2+a, 3 / 2+a$ or $1+a$.

(c) $\sum_{\mathscr{H}} \varrho_{H}=\sigma_{G^{-}}=-\omega_{G}+a$.

The subsequent Proposition collects the knowledge we have gained about the limit $\varepsilon \rightarrow 0$.

Proposition 1. The dimensionally regularized Feynman amplitude $\mathscr{T}_{G, \varepsilon}(p, n)$ can be decomposed into a sum of integrals of the form

$$
c_{n} \delta\left(\sum p_{i}\right) \int_{0}^{1} \prod_{H \neq G}\left(d t_{H} / t_{H}\right) t_{H}^{\nu h_{H}-\omega_{H}-\sigma_{H}} \int_{0}^{1} \prod^{\prime} d \beta_{\ell} f_{\varepsilon}(\underline{p}, \underline{\underline{\beta}}, \underline{\beta}, n) .
$$

Considered as distributions over $\mathscr{S}\left(\mathbb{R}^{4 K}\right)$ these integrals define meromorphic functions of $n$ by analytic continuation. In a certain finite neighbourhood of $n=4$ $\mathscr{T}_{G}(\underline{p}, n)=\lim _{\varepsilon \rightarrow 0} \mathscr{T}_{G, \varepsilon}(\underline{p}, n)$ exists in $\mathscr{S}^{\prime}\left(\mathbb{R}^{4 K}\right)$ and is meromorphic in $n . \mathscr{T}_{G, \varepsilon}(\underline{p}, n)$ as well as $\mathscr{T}_{G}(p, n)$ has a pole at $n=4$ resulting only from $t_{H^{-i n t e g r a t i o n s ~ c o r r e s p o n d i n g ~}}$ to divergent 1 PI subgraphs $H \in \mathscr{F}$.

Proof. The meromorphy of $\mathscr{T}_{G, \varepsilon}(p, n)$ is shown as usual, considering the powers $t_{H}^{v h_{H}-\omega_{H}-\sigma_{H}-1}$ as distributions [2]. The preceding lemma in combination with the $\varepsilon$-Lemma allows to take the limit $\varepsilon \rightarrow 0$ for $|n-4|<\min (a, 1-a) / h_{G}$ without changing the pole structure. Two facts are responsible for that:

i) $-\omega_{H}-\sigma_{H} \geqq 1-a>0$ for all $H \in \mathscr{C}_{\infty}$ if there exists some $H^{-}$; if $\mathrm{H}^{-}$does not exist, $\sigma_{H}=0$ and $H$ is $1 P I$;

ii) $\lim _{\varepsilon \rightarrow 0} f_{\varepsilon}(\underline{p}, \underline{t}, \underline{\beta}, n)$ considered as an element of $\mathscr{S}^{\prime}\left(\mathbb{R}^{4(K-1)}\right)$ is continuos in $(t, \underline{\beta})$ in the integration region and even $C^{\infty}$ there in all $t_{H}$ 's for which $\sigma_{H}=0$, due to Lemma $7^{2}$.

\section{III.3. Non-renormalizable Vertices}

For the formulation of the Action Principle in its general form as established for massive theories in [1] we have to relax the condition of strict renormalizability. From now on we treat arbitrary graphs $G$ without super-renormalizable $\left(\delta_{a}^{G}<4\right)$ internal vertices, i.e. we allow arbitrary external vertices, but only internal vertices of dimension $\delta_{a}^{G} \geqq 4$. This restriction is easily seen (up to one internal vertex of dimension three, discussed in Section II.4) to be in general necessary for the existence of Green's functions as distributions.

The only complication compared to the case already treated is due to the fact that the inequality $\omega_{H}<0$ for all $H \in \mathscr{H}$ ceases to be true. That can give rise to seemingly non-integrable singularities in the $t_{H}$-integrals for some $H \in \mathscr{H}$. These new singularities, however, are only apparent and could be avoided by a different sector division, namely the one employed in the massive case. Yet, there is a simple trick to avoid that complication altogether. By hanging onto the external vertices of $G$ suitable new legs we enforce $\omega_{H}<0$ for all subgraphs containing external vertices.

2 This differentiability is needed in order to perform the analytic continuation and to display the poles at $n=4$ 
For any graph $G$ we construct a new one, $\bar{G}$, in the following way: We take any one of the external vertices $V_{a}$ of $G$. If $\delta_{a}^{G} \geqq 3$ we add one more vertex $\left(n_{a}=1\right)$ and join it to $V_{a}$ by one single line with propagator $k_{G} ! /\left[i\left(m^{2}-p^{2}\right)+0\right]^{k_{G}+1}$ with some integer $k_{G}$. In case $\delta_{a}^{G}<3$ we add $n_{a}=4-\left[\delta_{a}^{G}\right]$ of these new vertices and lines. Having done that for all external vertices of $G$ the graph $\bar{G}$ results. To the vertices of $\bar{G}$ we assign new dimensions $\delta_{a}^{\bar{G}}$ in the following way: If $V_{a}$ is an internal vertex of $G$, we put $\delta_{a}^{\bar{G}}=\delta_{a}^{G}$; if $V_{a}$ is an external vertex of $G$ we put $\delta_{a}^{\bar{G}}=\delta_{a}^{G}+n_{a} \geqq 4$; finally if $V_{a}$ is one of the newly added vertices, i.e. an external vertex of $\bar{G}$, we put $\delta_{a}^{\bar{G}}=1-2 k_{G}$. We choose $k_{G}>\omega_{\bar{G}}^{\text {int }} / 4$.

With these definitions $\bar{G}$ has the following properties:

(a) $\delta_{a}^{\bar{G}} \geqq 4$ for all internal vertices $V_{a}$ of $\bar{G}$;

(b) all external vertices of $\bar{G}$ are joined to the rest of the graph by exactly one line. Let us call the set of these "external lines" $\mathscr{L}_{\text {ext }}$;

(c) $\omega_{H}<0$ for all connected graphs $H \subset \bar{G}$ containing at least two external vertices of $\bar{G}$. This is guaranteed by our choice of $k_{G}$.

Clearly the amplitude for $G$ can be recovered from that one for $\bar{G}$ by amputation of the external lines, i.e. by multiplication with a suitable polynomial in the external momenta. For $\varepsilon>0 \mathscr{T}_{\boldsymbol{G}, \varepsilon}$ is independent of $m, k_{\boldsymbol{G}}$ and the additional momenta introduced for all vertices with $n_{a}>1$; if the limit $\varepsilon \rightarrow 0$ exists for $\mathscr{T}_{\bar{G}, \varepsilon}$, and therefore also for $\mathscr{T}_{G, \varepsilon}$, then $\mathscr{T}_{G}$ is independent of $m, k_{G}$ and the additional momenta as well. From now on we treat only graphs of the type of $\bar{G}$ and denote them again by $G$ for simplicity.

Using the Feynman parameter representation

$$
k_{G} ! /\left[i\left(m^{2}-p^{2}\right)+0\right]^{k_{G}+1}=\lim _{\varepsilon \rightarrow 0} \int_{0}^{\infty} d \alpha \alpha^{k_{G}} e^{i \alpha\left(p^{2}-m^{2}+i \varepsilon\right)}
$$

for the external lines, the integral corresponding to Equation (8) reads

$$
\begin{gathered}
\Gamma\left(\left(v h_{G}-\omega_{G}+r\right) / 2\right) \int_{0}^{1} \prod_{H \neq G}\left(d t_{H} / t_{H}\right) t_{H}^{v h_{H}-\omega_{H}} \int_{0}^{1} \prod^{\prime} d \beta_{\ell} \prod_{\mathscr{L}_{\mathrm{ext}}} \beta_{\ell}^{k_{G}} g_{r}(\underline{p}, \underline{\underline{\mathrm{e}}}, \underline{\beta}, n) \\
\cdot\left[-i \hat{V}(\underline{p}, \underline{t}, \underline{\beta})+i m^{2} \sum_{\mathscr{L}_{\mathrm{ext}}} \beta_{\ell} \eta_{H}^{2}+\varepsilon \sum_{\mathscr{L}_{G}} \beta_{\ell} \eta_{H_{\ell}}^{2}\right]^{\left(\omega_{G}-v h_{G}-r\right) / 2} .
\end{gathered}
$$

Again having in mind the $\varepsilon$-Lemma we rewrite this in the form

$$
\begin{aligned}
& \Gamma\left(\left(v h_{G}-\omega_{G}+r\right) / 2\right) \int_{0}^{1} \prod_{H \neq G}\left(d t_{H} / t_{H}\right) t_{H}^{\nu h_{H}-\omega_{H}-\sigma_{H}} \int_{0}^{1} \prod^{\prime} d \beta_{\ell} h(t, \underline{\beta}, n) \\
& \cdot Q\left(\eta_{H} q_{H}\right) \prod_{\mathscr{H}} \eta_{H}^{O_{H}} \prod_{\mathscr{L} \text { ext }}\left(\beta_{\ell} \eta_{H_{\ell}}^{2}\right)^{k_{\ell}}\left[-i d\left(\eta_{H} q_{H}\right)+i m^{2} \sum_{\mathscr{L} \mathrm{ext}} \beta_{\ell} \eta_{H}^{2}+\varepsilon\right]^{-\left(v h_{G}-\omega_{G}+r\right) / 2}
\end{aligned}
$$

where $H_{\ell}$ is the smallest member of $\mathscr{C}_{\infty}$ (actually a member of $\mathscr{H}$ ) containing line $\ell$. We put all $k_{\ell}=k_{G}$, except when $H_{\ell}$ is the minimal element of $\mathscr{H}$, in which case we put $k_{\ell}=k_{G}-\omega_{G}^{\text {int }} / 4>0$.

For the definitions of the $\sigma_{H}$ 's we slightly modify the formula in Equation (17) to

$$
\sigma_{H}=\max \left\{0,\left[-\omega_{G}^{\mathrm{int}}+\sum_{i}\left(a-\omega_{H_{i}, G}^{\mathrm{ext}}\right)\right]\right\}
$$


where now according to our definition

$$
\omega_{H_{i}, G}^{\mathrm{ext}}=4-K_{H_{i}}\left(2 k_{G}+3\right)
$$

if $H_{i}$ has $K_{H_{i}}$ of the external vertices of $G$. Again we notice that $\sigma_{H}=0$ if there is no $H^{-}$. The $\varrho_{H}$ 's corresponding to these $\sigma_{H}$ 's are given by

$$
\varrho_{H}=\left\{\begin{array}{l}
\sigma_{H}+\omega_{G}^{\mathrm{int}}-4 k_{G} \text { if there is no } H_{-} ; \\
\sigma_{H}-\sigma_{H_{-}}-2\left(K_{H}-K_{H_{-}}\right) k_{G} \text { else } .
\end{array}\right.
$$

Going again through the different possibilities for $H_{-}$as before one proves the analogue of Lemma 2, namely

Lemma 3. (a) $-\omega_{H}-\sigma_{H} \geqq 1-$ a for all $H \in \mathscr{C}_{\infty}, H \neq G$, which contain some $H^{\prime} \in \mathscr{H}$ (i.e. if $\mathrm{H}^{-}$exists); otherwise $\sigma_{H}=0$;

(b) the $\varrho_{H}$ 's obey $0<\varrho_{H} \leqq 4-a$;

(c) $k_{\ell}>0$ for all $\ell \in \mathscr{L}_{\text {ext }}$;

(d) $\sum_{\mathscr{H}} \varrho_{H}+2 \sum_{\mathscr{L} \text { ext }} k_{\ell}+r=-\omega_{G}+r+a$.

With the information contained in Lemma 3 we are ready to apply the $\varepsilon$-Lemma and prove the validity of Proposition 1 also in the case under discussion.

Up to now we have assumed that the graph $G$ contains no tadpoles. In the following we will show that $\lim _{\varepsilon \rightarrow 0} \mathscr{T}_{G, \varepsilon}$ vanishes whenever $G$ contains tadpoles.

Let $G$ be a graph with tadpoles and $G^{0}$ the graph obtained from $G$ by amputation of all the tadpoles (and the lines connecting them with $G^{0}$ ). Every tadpole $G_{i}$ is connected to $G^{0}$ by a massless boson line with zero momentum and has superficial degree of divergence $\omega_{G_{\imath}} \geqq 3$. The amplitude for $G_{i}$ is

$$
\mathscr{T}_{G_{\imath}, \varepsilon}\left(p_{i}\right)=c_{i} \delta\left(p_{i}\right) \varepsilon^{v h_{G_{i}}+\omega_{G_{i}}}
$$

(and vanishes if $\omega_{G_{i}}$ is odd). $G^{0}$ has a number of super-renormalizable internal vertices which reduce $\omega_{G^{\circ}}$ by one for every tadpole attached to it. This is, however, overcompensated by the factor $c_{i} \varepsilon^{\nu h_{G_{t}}+\omega_{G_{i}}-1}$ supplied by each tadpole (together with the connecting line).

A slight modification of the $\varepsilon$-Lemma (compare Appendix B) ensures that

$$
\lim _{\varepsilon \rightarrow 0} \mathscr{T}_{G, \varepsilon}=\lim _{\varepsilon \rightarrow 0} \mathscr{T}_{G^{0}, \varepsilon} \prod_{i} c_{i} \varepsilon^{\nu h_{G_{i}}+\omega_{G_{i}}-1}=0,
$$

i.e. the amplitude for all graphs which contain tadpoles vanishes in the limit $\varepsilon \rightarrow 0$.

\section{II.4. Green's Functions with One Internal Vertex of Dimension 3}

For the formulation of normalization conditions in gauge theories it is useful to define Green's functions with one external momentum (attached to a vertex $V_{a}$ with dimension $\delta_{a}^{G}=3$ ) equal to zero; this is possible whenever the graph $G$ is $1 P I$ with respect to $V_{a}$ which becomes an internal vertex by this procedure. In order to show that such Green's functions exist, we have to estimate the degrees and to choose the $\sigma_{H}$ 's slightly more carefully than in the previous sections. 
Definition. We split the degree $\omega_{G}$ into the internal degree of $G$

$$
\omega_{G}^{\mathrm{int}}=-\sum_{\mathscr{J}_{G}}\left(4-\delta_{a}^{G}\right) \geqq 0 ; \quad \mathscr{J}_{G}=\left\{V_{a} \in \mathscr{V}_{G} \mid \mathscr{U}_{G}: \delta_{a}^{G} \geqq 4\right\} ;
$$

the super-renormalizable degree of $G$

$$
\omega_{G}^{s r}=-\sum_{\mathscr{K}_{G}}\left(4-\delta_{a}^{G}\right) \leqq 0 ; \quad \mathscr{K}_{G}=\left\{V_{a} \in \mathscr{V}_{G} \backslash \mathscr{U}_{G}: \delta_{a}^{G}<4\right\} ;
$$

and the external degree of $G$

$$
\omega_{G}^{\mathrm{ext}}=\omega_{G}-\omega_{G}^{\mathrm{int}}-\omega_{G}^{\mathrm{sr}} \text {. }
$$

For any $H \subset G$ we define the relative super-renormalizable degree of $H$ in $G$ by

$$
\omega_{H, G}^{s r}=-\sum_{\mathscr{K}_{G} \cap \mathscr{V}_{H}}\left(4-\delta_{a}^{G}\right) \text {. }
$$

We only consider graphs $G$ with $\omega_{G}^{s r}>-2$ and assume that for every $1 P I$ tadpole $G_{i} \subset G \omega_{G_{i}, G}^{\text {sr }}>-1$ holds. As in Section II. 3 we construct a new graph $\bar{G}$ and call it again $G$. Due to our assumption $\omega_{G_{l}, G}^{\text {sr }}>-1$ the amplitudes for graphs with tadpoles vanish in the limit $\varepsilon \rightarrow 0$.

Let $G$ be a graph of the type of $\bar{G}$ without tadpoles and $\mathscr{C}_{\infty}$ a $c_{\infty}$-family for $G$; for any $H \in \mathscr{C}_{\infty}$ with $c$-components $H_{i}$ we choose

$$
\sigma_{H}=\max \left\{0,\left[-\omega_{G}^{\mathrm{int}}-\max _{H^{\prime}} \omega_{H^{\prime}, G}^{\mathrm{si}} / 2+\sum_{i}\left(a-\omega_{H_{i}, G}^{\mathrm{ext}}\right)\right]\right\}
$$

where $\max _{H^{\prime}}$ is to be taken over all $H^{\prime} \in \mathscr{C}_{\infty}$ with $\mu_{H^{\prime}}=\mu_{H}$. Once more $\sigma_{H}=0$ if there is no $H^{-}$. The corresponding $\varrho_{H}$ 's are given by Equation (26). Choosing $-\omega_{G}^{\text {sr }} / 2<$ $a<1$ and proceeding further as in Section II.3, we verify Lemma 3 with two changes:

(b') $0<\varrho_{H}<4-\left(a+\omega_{G}^{\mathrm{sr}} / 2\right)<4$;

(d') $\sum_{\mathscr{H}} \varrho_{H}+2 \sum_{\mathscr{P}_{\mathrm{ext}}} k_{\ell}+r=-\omega_{G}+r+\left(a+\omega_{G}^{\mathrm{sr}} / 2\right)>-\omega_{G}+r$.

To prove Lemma 3 (a) we have used that

$$
\omega_{H} \leqq \omega_{G}^{\mathrm{int}}-1+\sum_{i}\left(\omega_{H_{i}, G}^{\mathrm{ext}}-1\right)
$$

whenever an $H^{\prime} \in \mathscr{C}_{\infty}$ with $H^{\prime} \underset{\ddagger}{\supsetneqq}, \mu_{H^{\prime}}=\mu_{H} \geqq 1$ exists; due to the construction of $c_{\infty}$-families, at least one $c$-component $H_{i}$ of $H$ is joined to the rest of $G$ by two or more lines.

As a consequence of Lemma 3 Proposition 1 remains true also in the case under discussion.

\section{Renormalization and the Action Principle}

The renormalization of the dimensionally regularized Feynman amplitudes $\mathscr{T}_{G}(\underline{p}, n)=\lim _{\varepsilon \rightarrow 0} \mathscr{T}_{G, \varepsilon}(\underline{p}, n)$, i.e. the elimination of the singularity of $\mathscr{T}_{G}(\underline{p}, n)$ at $n=4$, works--thanks to Proposition 1-exactly like for massive theories as outlined in Ref. [1]. 
Recursively in the number of loops (powers of $\hbar$ ) we construct for each $1 P I$ graph $G$ the amplitude $\overline{\mathscr{T}}_{G, \varepsilon}(p, n)$ containing already all counter terms corresponding to $1 P I$ subgraphs of $G$ with fewer loops than $G$. The left over singularity at $n=4$ - present only if $\omega_{G} \geqq 0$-is shown to be a homogeneous polynomial in the external momenta of $G$ and $\sqrt{\varepsilon}$. This pole term represents the counter term corresponding to $G$ in the construction of $\overline{\mathscr{T}}_{G^{\prime}, \varepsilon}(p, n)$ for all $G^{\prime}$ with more loops than $G$. If we denote by $\mathscr{R}_{G, \varepsilon}(p, n)$ the regular part of the Laurant series expansion of $\overline{\mathscr{T}}_{G, \varepsilon}(p, n)$ around $n=4$ then $\mathscr{R}_{G}(p, n)=\lim _{\varepsilon \rightarrow 0} \mathscr{R}_{G, \varepsilon}(p, n)$ resp. its value at $n=4$ is defined to be the dimensionally renormalized amplitude for $G$. In order to construct $\mathscr{R}_{G}(p, n)$ also for $1 P R$ graphs it is sufficient to take counter terms for all their $1 P I$ subgraphs into account.

Let us state the result as

Theorem 1. For any Lagrangean without super-renormalizable couplings describing the interaction of massless particles dimensionally renormalized Green's functions for arbitrary compositive field operators can be defined in every order of perturbation theory as distributions over $\mathscr{S}$. The renormalization is achieved by adding to the Lagrangean local counter terms of minimal degree according to Weinberg's power counting having poles at $n=4$.

All renormalization parts with positive superficial degree of divergence $\omega \geqq 1$ vanish at the origin in momentum space up to order $\omega-1$.

Proof. The statement about the structure of counter terms for $\varepsilon>0$ is proven exactly like for massive theories as outlined in Ref. [1] and need not be repeated here. Let us just remark that $\varepsilon$, as we have used it, acts like a mass term.

The counter terms necessary for renormalization (for $\varepsilon>0$ ) are again of the strictly- or non-renormalizable type $(\delta \geqq 4)$, except when they are proportional to $\varepsilon$ resp. $\varepsilon^{2}$; in this case $\delta \geqq 2$ resp. $\delta \geqq 0$. The argument used for the contributions from tadpoles (end of Section II.3) applies also here and guarantees that such contributions vanish in the limit $\varepsilon \rightarrow 0$. The same argument ensures that the unwanted contributions proportional to $\varepsilon$ in the equations of motion, coming from $\left(\square+m^{2}-i \varepsilon\right)$, vanish in the limit.

The renormalized amplitude $\mathscr{R}_{G, \varepsilon}$ (for $n \neq 4$ ) is a finite sum of the unrenormalized, regularized amplitude + counter terms, each of which is continuous in $\mathscr{S}^{\prime}\left(\mathbb{R}^{4 K}\right)$ for $\varepsilon \geqq 0$. Therefore $\mathscr{R}_{G}$ exists in $\mathscr{S}^{\prime}$. Since $\mathscr{R}_{G, \varepsilon}$ is analytic at $n=4, \mathscr{R}_{G}$ can also be continued to $n=4$.

The vanishing at the origin of momentum space up to order $\omega-1$ for all renormalization parts with superficial degree of divergence $\omega \geqq 1$ is a consequence of the homogeneity properties of $\mathscr{T}_{G}(p, n)$ for $n \neq 4$.

The important result, that Schwinger's Action Principle holds without modifications due to radiative corrections proven for massive theories in [1] remains also true here. Let us shortly repeat the formulation given in [1]. We assume that the Lagrangean $\mathscr{L}$ depends on quantized fields $\phi$, external fields $\underline{a}$ and space-time independent parameters $\underline{\lambda}$. By $Z(\underline{a}, \underline{\lambda})$ we denote the connected vacuum expectation value of the perturbation series of the scattering operator corresponding to $\mathscr{L}$, i.e.

$$
Z(\underline{a}, \underline{\lambda})=\hbar\left\langle T \exp i \hbar^{-1} \int \mathscr{L}_{\text {int }}(\phi(x), \underline{a}(x), \underline{\lambda}) d x\right\rangle_{c}^{0} .
$$


Schwinger's Action Principle says:

i) local variations of the quantized fields $\phi$ of the form $\delta \phi(x)=\underline{P}(\phi(x)) \delta \varepsilon(x)$ with some polynomials $\underline{P}$ leave $Z$ invariant, i.e.

$$
0=\left\langle T \delta \mathscr{L} \exp i \hbar^{-1} \int \mathscr{L}_{\text {int }}(x) d x\right\rangle_{c}^{0}
$$

where

$$
\delta \mathscr{L}=\int \underline{P}(\phi(x))\left(\delta \mathscr{L} / \delta \phi(x)-\partial_{\mu} \delta \mathscr{L} / \delta \partial_{\mu} \phi(x)\right) \delta \varepsilon(x) d x
$$

ii) variations of the external fields resp. parameters result in

$$
-i \delta Z / \delta \underline{a}(x)=\left\langle T \delta \mathscr{L} / \delta \underline{a}(x) \exp i \hbar^{-1} \int \mathscr{L}_{\text {int }}(x) d x\right\rangle_{c}^{0},
$$

resp.

$$
-i \partial Z / \partial \underline{\lambda}=\left\langle T \partial \mathscr{L} / \partial \underline{\lambda} \exp i \hbar^{-1} \int \mathscr{L}_{\text {int }}(x) d x\right\rangle_{c}^{0} .
$$

Theorem 2. Schwinger's Action Principle as described above holds for the renormalized functional $Z(\underline{a}, \underline{\lambda})$, if there is no explicit $n$-dependence involved.

Proof. Exactly like in the massive case.

Remark. For the discussion of anomalies arising from an explicit $n$-dependence of the variation $\delta \mathscr{L}$ we refer to [1].

\section{Appendix A}

In this appendix we have collected some information about Feynman graphs which is needed to prove the existence of $\mathscr{T}_{G}=\lim _{\varepsilon \rightarrow 0} \mathscr{T}_{G, \varepsilon}$. We refer to Appendix A of [1] for basic definitions.

Let $G$ be a connected graph with vertices $\mathscr{V}_{G}=\left\{V_{i}, i=1, \ldots, M\right\}$. We divide them into $K$ external and $M-K$ internal vertices. The auxiliary momenta associated with the internal vertices will eventually be set to zero, the external momenta will be kept variable and we have to justify the interpretation of $\mathscr{T}_{G}$ as a distribution over $\mathscr{S}\left(\mathbb{R}^{4 K}\right)$. Let $\mathscr{U}_{G}=\left\{V_{i}, i=1, \ldots, K\right\}$ be the set of all external vertices. For any subgraph $H \subset G$ we define $\mathscr{U}_{H}=\mathscr{U}_{G} \cap \mathscr{V}_{H}$ and $K_{H}=\left|\mathscr{U}_{H}\right|$.

A tadpole is a connected subgraph $H \subset G$ with $\mathscr{U}_{H}=\emptyset$ which is connected to the rest of $G$ by exactly one line. In the following we assume that $G$ contains no tadpoles, $K \geqq 2$ and that exactly one line of $G$ is attached to each external vertex.

For any subgraph $H \subset G$ we construct a graph $H_{\infty}$ by identifying all its external vertices. A $c_{\infty}$-family $\mathscr{C}_{\infty}$ for $G$ is a set of subgraphs $H_{i} \subset G$ (without trivial $c$-components) such that $\mathscr{C}=\left\{H_{i \infty}\right\}$ is a maximal forest for $G$. A labelled $c_{\infty}$-family is a pair $\left(\mathscr{C}_{\infty}, \sigma\right)$ such that $(\mathscr{C}, \sigma)$ is a labelled forest. For any $H \in \mathscr{C}_{\infty}$ let $\mathscr{M}(H)$ be the set of all maximal genuine subgraphs of $H$ which are contained in $\mathscr{C}_{\infty}$. For any $\ell \in \mathscr{L}_{G}$ let $H_{\ell}$ be the smallest member of $\mathscr{C}_{\infty}$ which contains $\ell$ (i.e. $\ell \in \mathscr{L}_{H / M(H)}$ ). Note. Our $c_{\infty}$-families differ from the $s_{\infty}$-families of Ref. [3] by the use of $1 P I$ subgraphs instead of irreducible ones.

Lemma 4. (a) Any $c_{\infty}$-family $\mathscr{C}_{\infty}$ for $G$ contains $G$;

(b) any $c_{\infty}$-family $\mathscr{C}_{\infty}$ for $G$ can be labelled; 
(c) the domains $\mathscr{D}\left(\mathscr{C}_{\infty}, \sigma\right)=\left\{\underline{\alpha}: 0 \leqq \alpha_{\ell} \leqq \alpha_{\sigma(H)}\right.$ for all $\left.\ell \in \mathscr{L}_{H}\right\}$ are a partition of $\alpha$-space $\left\{\underline{\alpha}: \alpha_{\ell} \geqq 0\right.$ for all $\left.\ell\right\}$ up to sets of measure zero;

(d) for any $H \in \mathscr{C}_{\infty} H / \mathscr{M}(H)$ has exactly one nontrivial c-component $\bar{H}$, which is either a tree $\left(h_{\bar{H}}=0\right)$ or 1 PI with one loop $\left(h_{\bar{H}}=1\right)$;

(e) $\mathscr{F}=\left\{H \in \mathscr{C}_{\infty}: \bar{H}\right.$ is $\left.1 P I\right\}$ has exactly $h_{G}$ elements; $T^{0}=G-\sigma(\mathscr{F})$ is a tree;

(f) $\mathscr{H}=\left\{H \in \mathscr{C}_{\infty}: \bar{H}\right.$ is a tree $\}$ has exactly $K-1$ elements which can be ordered by inclusion. The same ordering is induced by the numbers $\mu_{H}=K_{H}$ - (number of c-components of $H$ ) which take all integers from 1 to $K-1$ as values. $\mu_{H}=1$ always implies $K_{H}=2$, if $\mu_{H}-\mu_{H^{\prime}}=1$ then $K_{H}-K_{H^{\prime}}$ equals either zero or one or two.

These statements can be proved along the lines of Refs. [1-3].

From now on we consider one particular labelled $c_{\infty}$-family $\left(\mathscr{C}_{\infty}, \sigma\right)$. For any $H \in \mathscr{C}_{\infty}$ we denote by $H_{-}$resp. $H^{-}$the largest $H^{\prime} \in \mathscr{H}$ with $H^{\prime} \subsetneq H$ resp. $H^{\prime} \subset H$ if such an $H^{\prime}$ exists.

We may assign a number $\mu_{H}$ to all $H \in \mathscr{C}_{\infty}$ by the formula of Lemma 4 (f). $H^{-}$exists iff $\mu_{H} \geqq 1$ and in this case $H^{-}$is the smallest $H^{\prime} \in \mathscr{C}_{\infty}$ with $\mu_{H^{\prime}}=\mu_{H^{\prime}}$.

Let $H$ be a member of $\mathscr{C}_{\infty}$. To any vertex $V_{a}$ of $\bar{H}$ we assign a momentum $q_{a}$ which is the sum of all $p_{i}$ associated with those vertices $V_{i}$ which are mapped to $V_{a}$ by $H \rightarrow H / \mathscr{M}(H)$. Some of these sums contain external momenta $p_{i}(i \leqq K)$, we call these momenta $q_{a}$ as well as their vertices $V_{a}$ external and the other ones auxiliary resp. internal.

Lemma 5. (a) For any $H \in \mathscr{H} \bar{H}$ has exactly two external vertices;

(b) for any $H \in \mathscr{F} \bar{H}$ has either exactly one external vertex (if $H_{-}$exists) or none (if $H_{-}$does not exists).

Let $g_{H}, H \in \mathscr{C}_{\infty}$ be the momenta associated with all but one of the vertices of $\bar{H}$, chosen such that for $H \in \mathscr{F}$ they are all auxiliary and for $H \in \mathscr{H}$ only the first one, $q_{H}$, is external. These new momenta $g=\left(q_{H}, H \in \mathscr{C}_{\infty}\right)$ are obtained from $p=\left(p_{1}, \ldots, \hat{p}_{K}, \ldots, p_{M}\right)$ by a linear transformation $R: q=R \underline{p}$. We decompose $\underline{u}=\left(u_{\ell}, \ell \in \mathscr{L}_{G}\right)$ in a similar way: $\underline{u}=\left(\underline{u}_{H}, H \in \mathscr{C}_{\infty}\right), \underline{u}_{H}=\left(u_{\ell}, \ell \in \mathscr{L}_{\vec{H}}\right)$.

Lemma 6. (a) $\operatorname{det} R= \pm 1$;

(b) $\operatorname{det} R^{\mathrm{ext}}= \pm 1$, where $R^{\mathrm{ext}}$ is the restriction of $R$ to $p^{\mathrm{ext}}=\left(p_{1}, \ldots, p_{\mathrm{K}-1}\right)$ and $q^{\text {ext }}=\left(q_{H}, H \in \mathscr{H}\right)$

(c) the transformed incidence matrix $e R^{+}=\left(e_{H H^{\prime}}, H, H^{\prime} \in \mathscr{C}_{\infty}\right)$ has the following block structure:

$$
e_{H H^{\prime}}\left\{\begin{array}{l}
=0 \text { if } H \supset H^{\prime} ; \\
\text { is the incidence matrix for } \bar{H} \text { if } H=H^{\prime} ; \\
\text { indicates how } H^{\prime} \text { is contained in } H \text { if } H \supsetneqq H^{\prime}
\end{array}\right.
$$

Proof. (a) Up to permutations $R$ is a triangular matrix with all digonal elements $=1$ and (b) the same holds true for $R^{\text {ext }}$. (c) Momentum conservation at each vertex is expressed by $\underline{p}+e^{+} \underline{k}=0$, where $\underline{k}=\left(k_{\ell}, \ell=1, \ldots, L\right)$ are the internal momenta occuring in the momentum space formulation of the amplitude. The block structure follows from $q+R e^{+} \underline{k}=0$.

Remark. $q^{\text {ext }}$ together with $q_{0}=\sum_{i=1}^{K} p_{i}$ form a momentum family in the sense of Ref. [6]. 
We may now rewrite the quadratic form $V(\underline{p}, \underline{u}, \underline{\alpha})$ in terms of $\tilde{q}=\left(g_{H} \zeta_{H}, H \in \mathscr{C}_{\infty}\right)$ and $\underline{\tilde{u}}=\left(\underline{u}_{H} / \zeta_{H}, H \in \mathscr{C}_{\infty}\right)$

$$
\begin{aligned}
& V(p, \underline{u}, \underline{\alpha})=\left(\tilde{g}^{+} \underline{\tilde{u}}^{+}\right) \tilde{M}^{-1}\left(\begin{array}{l}
\tilde{g} \\
\tilde{u}
\end{array}\right) \\
& \text { with } \tilde{M}=\left(\begin{array}{cc}
0 & -2 \tilde{e}^{+} \\
-2 \tilde{e} & -4 \beta
\end{array}\right), \tilde{e}=\left(e_{H H^{\prime}} \zeta_{H^{\prime}} / \zeta_{H}, H, H^{\prime} \in \mathscr{C}_{\infty}\right) ;
\end{aligned}
$$

where $\beta$ is the diagonal matrix with elements $\beta_{\ell}=\alpha_{\ell} / \zeta_{H_{\ell}}^{2}$ : In addition $\operatorname{det} M=$ $\operatorname{det} \tilde{M} \prod_{\mathscr{F}} \zeta_{H}^{2}$.

Lemma 7. (a) $\tilde{M}$ is independent of $t_{G}$ and bounded in $\mathscr{D}$;

(b) $\operatorname{det} \tilde{M} \geqq 1$ in $\mathscr{D}$;

(c) the matrix $d=\left(d_{H H^{\prime}}, H, H^{\prime} \in \mathscr{H}\right)$ given by $V\left(p^{\mathrm{ext}}, \underline{\alpha}\right)=\sum_{H H^{\prime} \in \mathscr{H}} \zeta_{H} \zeta_{H^{\prime}} q_{H} q_{H^{\prime}} d_{H H^{\prime}}$ is positive definite in $\mathscr{D}$;

(d) $\tilde{M}^{-1}$ and $\operatorname{det} \tilde{M}$ are $C^{\infty}$ in $(t, \underline{\beta})$ from $\mathscr{D}$.

Proof. (a) Follows from $e_{H H^{\prime}}=0$ for $H \downarrow H^{\prime}$ and $\beta_{\ell} \leqq 1$ for all $\ell$.

(b) $\operatorname{det} M=\sum_{T} \prod_{\ell \notin \mathscr{T}_{T}} \alpha_{\ell} \geqq \prod_{\ell \notin \mathscr{L}_{T^{0}}} \alpha_{\ell}=\prod_{\mathscr{F}} \zeta_{H}^{2}$ where the sum runs over all trees in $G$.

(c) Following an argument of Ref. [3] we see that

$$
\begin{aligned}
\operatorname{det} M d(\underline{x}) & =\operatorname{det} M \sum_{H H^{\prime}} d_{H H^{\prime}} x_{H} x_{H^{\prime}}=\sum_{T_{2}} y_{T_{2}}^{2} \prod_{\ell \in \mathscr{L}_{T_{2}}} \alpha_{\ell} \\
& \geqq\left[\sum_{\mathscr{H}}\left(y_{T_{2}(H)} \zeta_{H}\right)^{2}\right]\left[\prod_{\mathscr{F}} \zeta_{H}^{2}\right]
\end{aligned}
$$

where the first sum runs over all 2-trees in $G$ and

$$
\zeta_{H} y_{T_{2}(H)}=x_{H}+\sum_{H^{\prime} \supsetneq H} c_{H H^{\prime}} x_{H^{\prime}} \zeta_{H} / \zeta_{H^{\prime}}
$$

with some coefficients $c_{H H^{\prime}}$. Therefore $d(\underline{x}) \geqq\left[\sum_{\mathscr{H}}\left(y_{T_{2}(H)} \zeta_{H}\right)^{2}\right] / \operatorname{det} \tilde{M}$ and this vanishes only if all $x_{H}$ are zero.

(d) This follows from $\tilde{M}$ being a polynomial in $(\underline{t}, \underline{\beta})$ and (b).

Lemma 8. The spin polynomials produced by the application of $Z(-i \partial / \partial u)$ to $\exp \left(-i V\left(p^{\mathrm{ext}}, \underline{u}, \underline{\alpha}\right)\right)$ are, apart from negative powers of $\zeta_{H}$ absorbed into $\prod_{\mathscr{C}_{\infty}} t_{H}^{-\omega_{H}}$, polynomials in $g$ with coefficients which are $C^{\infty}$ in $(t, \beta)$.

Proof. This follows directly from the representation (1) and Lemma 7 (d).

\section{Appendix B. Proof of the $\varepsilon$-Lemma}

For $\operatorname{Re}(\varrho) \leqq 0$ the right hand side of Equation (II.10) converges pointwise to a continuous polynomially bounded and locally integrable function. In the following we assume $\operatorname{Re}(\varrho)>0$. 
We first use Jacobi's diagonalization procedure to determine a linear transformation $S: q \rightarrow q^{\prime}=S g$ [3] such that $S_{i j}=0$ for $i>j$ and $d\left(\eta_{j} q_{j}\right)=\sum_{j} \eta_{j}^{2} q_{j}^{\prime 2} . S$ and $S^{-1}$ are $C^{\infty}$ in $\left(\eta_{j}, d_{i j}\right)$ and $Q^{\prime}\left(q^{\prime}\right)=Q(q)$ is again a homogeneous polynomial with coefficients which are $C^{\infty}$ in $\eta_{j}$ and in the original coefficients. For simplicity we write again $q$ instead of $q^{\prime}$ and assume $Q^{\prime}(\underline{q})$ to be a product of monomials $Q_{j}\left(q_{j}\right)$ of degree $r_{j}\left(\sum_{j} r_{j}=r\right)$.

Let $Q(q)$ be a monomial of degree $r$ in $q \in \mathbb{R}^{4}$. Defining $Q(q) /\left(-i q^{2}+0\right)^{s}$ for $0<\operatorname{Re}(s)<2+r / 2$ as usual [4], the following estimate can be derived for all $k=0,1, \ldots$ and $\varphi \in \mathscr{S}\left(\mathbb{R}^{4}\right)[3]$

$$
\begin{aligned}
\left|\left\langle\Gamma(s) Q(q)\left(-i q^{2}+0\right)^{-s}, \varphi\right\rangle\right| \leqq & C_{k}(1+|\operatorname{Im} s|)^{\operatorname{Re} s-\frac{1}{2}-2 k} \\
& \cdot \sup _{q \in \mathbb{R}^{4}}\left|(1+\|q\|)^{2 k+5} Q(q) \square \square^{k} \varphi(q)\right|
\end{aligned}
$$

where $\|q\|$ is the euclidean norm of $q$. In addition we estimate for $s \neq 0,-1,-2, \ldots$ and $\operatorname{Re}(z) \geqq 0$

$$
\left|\Gamma(s) / z^{s}\right| \leqq C(1+|\operatorname{Im} s|)^{\operatorname{Re} s-\frac{1}{2}}|z|^{-\operatorname{Re} s} \exp \left(-\frac{1}{2} \pi|\operatorname{Im} s|+\arg z \operatorname{Im} s\right) .
$$

That permits us to use the following Mellin-integral representation $\left(\eta_{j}>0, \gamma_{t}>0\right)$ (we set $s_{J+L+1}=0$ ):

$$
\begin{aligned}
f(\varepsilon)= & \prod \eta_{j}^{\rho_{j}} Q_{j}\left(\eta_{j} q_{j}\right) \prod \gamma_{\ell}^{k_{\ell}}\left(-i \sum \eta_{j}^{2} q_{j}^{2}+i \sum \gamma_{\ell} \chi_{\ell}+\varepsilon\right)^{-\varrho / 2} \\
= & \Gamma(\varrho / 2)^{-1} \int_{\sigma_{1}^{\prime}-i \infty}^{\sigma_{1}^{\prime}+i \infty}(2 \pi i)^{-1} d s_{1} \ldots \int_{\sigma_{J+L}^{\prime}-i \infty}^{\sigma_{J}^{\prime}+L+i \infty}(2 \pi i)^{-1} d s_{J+L} \Gamma\left(\varrho / 2-s_{1}\right) \varepsilon^{s_{1}-\varrho / 2} \\
& \cdot \prod_{j=1}^{J} \Gamma\left(s_{j}-s_{j+1}\right) \eta_{j}^{\varrho_{j}} Q_{j}\left(\eta_{j} q_{j}\right)\left(-i \eta_{j}^{2} q_{j}^{2}+0\right)^{s_{j+1}-s_{J}} \\
& \cdot \prod_{l=1}^{L} \Gamma\left(s_{J+\ell}-s_{J+\ell+1}\right) \gamma_{\ell}^{k_{\ell}}\left(i \gamma_{\ell} \chi_{\ell}\right)^{S_{J+\ell+1}-s_{J+\ell}}
\end{aligned}
$$

for $\operatorname{Re}(\varrho / 2)>\sigma_{1}^{\prime}>\ldots>\sigma_{J+L}^{\prime}>0$ and $\sigma_{j}^{\prime}-\sigma_{j+1}^{\prime}<\left(\varrho_{j}+r_{j}\right) / 2, j=1, \ldots, J$. The integral is absolutely convergent (in $\mathscr{S}^{\prime}$ ) due to the above estimates. Now we stretch the distances between the contours, shifting part of them to the left, part to the right, always respecting the condition $\operatorname{Re}\left(s_{j}-s_{j+1}\right)<\left(\varrho_{j}+r_{j}\right) / 2$ such that $\operatorname{Re}\left(s_{1}-\varrho / 2\right)$ becomes slightly positive and $\operatorname{Re}\left(s_{J+\ell}-s_{J+\ell+1}\right)$ becomes less than $k_{\ell}$ (for $\ell=1, \ldots, L)$. The possibility to do so is due to the assumption $\operatorname{Re}(\varrho)<\sum \varrho_{j}+$ $2 \sum k_{\ell}+r$. From crossing the pole of $\Gamma\left(\varrho / 2-s_{1}\right)$ at $s_{1}=\varrho / 2$ we pick up a residue which does not depend on $\varepsilon$, whereas the remaining integral vanishes in the limit $\varepsilon \rightarrow 0$. That proves the existence of $\lim _{\varepsilon \rightarrow 0} f(\varepsilon)$. Due to the factors $\eta_{j}^{\rho_{j}}$ resp. $\gamma_{\ell}^{k_{\ell}}$ the whole expression is obviously continuous in the $\eta_{j}$ 's resp. $\gamma_{\ell}$ 's even down to $\eta_{j}=\gamma_{\ell}=0$.

Differentiability with respect to the coefficients of $Q$ and $d$ follows from the fact that such differentiations reproduce the form of Equation (II.10). 
Remark. The following generalization-needed at various places-is also seen to hold:

$$
\lim _{\varepsilon \rightarrow 0} \varepsilon^{k} Q\left(\eta_{j} q_{j}\right) \prod \eta_{j}^{o_{j}} \prod \gamma_{\ell}^{k_{\ell}}\left(-i d\left(\eta_{j} q_{j}\right)+i \sum \gamma_{\ell} \chi_{\ell}+\varepsilon\right)^{-\varrho / 2-k}=0 \text { for } \operatorname{Re}(k)>0
$$

We just shift the contours as before, this time without crossing the pole of $\Gamma\left(\varrho / 2+k-s_{1}\right)$.

\section{References}

1. Breitenlohner, P., Maison, D.: Dimensional renormalization and the action principle. Commun. math. Phys. 52, 11-38 (1977)

2. Speer,E.R.: Generalized Feynman amplitudes. Princeton: Princeton University Press 1969

3. Pohlmeyer,K.: Large momentum behaviour of the Feynman amplitudes in the $\phi_{4}^{4}$-theory. In: International Symposium on Mathematical Problems in Theoretical Physics (H. Araki, ed.). Berlin-Heidelberg-New York: Springer 1975

4. Gelfand, I. M., Shilov, G.E.: Generalized functions, Vol. I. New York: Academic Press 1964

5. Zimmermann, W.: Lectures on elementary particles and quantum field theory (S. Deser, M. Grisaru, H. Pendleton, eds.), Vol. I, p. 397. Cambridge: M.I.T. Press 1970

Lowenstein, J.H.: Seminars on renormalization theory, Vol. I. Maryland Technical Report 73-068 (1972)

6. Trute,H.: Graphical rules for the diagonalization of the Feynman denominator. Preprint DESY 74/44 September 1974

Communicated by K. Symanzik

Received July 28, 1975; in revised form January 8, 1976 